\title{
Topology regulates pattern formation capacity of binary cellular automata on graphs
}

\author{
Carsten Marr ${ }^{\dagger}$ and Marc-Thorsten Hütt ${ }^{\ddagger}$ \\ Bioinformatics Group, Department of Biology, Darmstadt University of Technology ${ }^{\S}$
}

\begin{abstract}
We study the effect of topology variation on the dynamic behavior of a system with local update rules. We implement one-dimensional binary cellular automata on graphs with various topologies by formulating two sets of degree-dependent rules, each containing a single parameter. We observe that changes in graph topology induce transitions between different dynamic domains (Wolfram classes) without a formal change in the update rule. Along with topological variations, we study the pattern formation capacities of regular, random, small-world and scale-free graphs. Pattern formation capacity is quantified in terms of two entropy measures, which for standard cellular automata allow a qualitative distinction between the four Wolfram classes. A mean-field model explains the dynamic behavior of random graphs. Implications for our understanding of information transport through complex, networkbased systems are discussed.
\end{abstract}

\section{Introduction}

Cellular automata (CAs) have been studied in terms of complexity theory and computational universality and, moreover, they often serve as models of pattern formation. The usual topologies of CAs are chains and regular lattices. Current research on applications of graph theory to the analysis of natural and technical systems reveals that many real systems are based on networks with a much more complex structure [1, 2, 3, 4, 5]. Small-world graphs and scale-free topologies are the epitome of the huge progress in attempts to assess observed structures and properly model evolutionarily expanding networks [6, 7, 8].

We, therefore, re-investigate a standard model system of spatiotemporal pattern formation, namely binary CAs, by implementing it on a graph and systematically varying topological features. How is the spatiotemporal pattern of a CA (for example the capacity to display oscillations or the complexity of an element's time evolution within the system) changed, when, e.g., some shortcuts are introduced into the system, or, the regular neighborhood structure is completely substituted by a random graph topology? While it is clear that the change of a specific CA rule alters the dynamics and, consequently, the

\footnotetext{
${ }^{\dagger}$ marr@bio.tu-darmstadt.de

†huett@bio.tu-darmstadt.de

${ }^{\S}$ Schnittspahnstr. 3-5, D-64287 Darmstadt, Germany
} 
spatiotemporal pattern, it seems worthwhile to study the changes under topology variation at a fixed update scheme. Some approaches already deal with binary dynamics on complex topologies. The Ising model for example has been implemented on a small-world graph [9] and the scale-free topology has served as the backbone for boolean dynamics [10], as well as for the SIS and SIR epidemic models [11, 12]. Only few attempts investigate the connection between changes in topology and the corresponding impact on CA dynamics. In [13] the "game of life" is studied on a small-world network and a phase transition at a critical network disorder is found. However, the link between dynamic features and topology variation, especially for one-dimensional CAs, has not been addressed yet. Therefore, in this paper we pursue the question, to what extent topology determines and constrains the capacity of a system to display certain forms of dynamics.

In the following, we briefly review the cellular automaton (CA) formalism and introduce the notation used throughout the paper.

A CA describes the deterministic time evolution of regularly coupled cells. Formally, a one-dimensional (1D) CA consists of a chain of $N$ cells in states $x \in \Sigma$, with the set of all possible cell states $\Sigma$, and a transition function $\Delta$. The standard topology of a 1D CA is a ring lattice where node $i$ is connected to its $k$ next neighbors ranging from $i-k / 2$ to $i+k / 2$ with even $k$. The transition function $\Delta$ maps the configuration $\tilde{\boldsymbol{y}}_{i}$ of cell state $i$ together with its $k$ neighboring cell states at time $t$, onto the state of the central cell $i$ in the next time step, $\Delta: \tilde{\boldsymbol{y}}_{i}(t)=\left\{x_{i-k / 2}(t), \ldots, x_{i}(t), \ldots, x_{i+k / 2}(t)\right\} \rightarrow x_{i}(t+1)$. For $s=|\Sigma|$ different cell states there exist $\xi=s^{k+1}$ different neighborhood configurations. Each transition function $\Delta$ maps these configurations on elements in $\Sigma$, resulting in $s^{\xi}$ possible transition functions. This combinatorial aspect of the set of possible transition functions is also often used to attribute rule numbers to the $s^{\xi}$ update rules. To this end, the neighborhood configurations $\tilde{\boldsymbol{y}}$ are ordered according to the increasing value of the corresponding binary number. In that way sorted, the list of mapped states represents the rule number in binary digits. In a system-wide picture, $\Delta$ defines the transitions between the $s^{N}$ different system configurations $\boldsymbol{x}=\left\{x_{1}, x_{2}, \ldots, x_{n}\right\}$. From this point of view, the configuration space together with the update rule $\Delta$ forms a directed network, where the transitions between the system's configurations are completely determined. After at most $s^{N}$ time steps, any finite-size CA consisting of $N$ elements revisits a previously encountered state and repeats its dynamics. This network-like aspect of CAs in configuration space is frequently discussed in studies of boolean dynamics.

We will restrict our investigation throughout this paper to a binary state space $\Sigma=\{0,1\}$, and update all cells synchronously at every time step. The minimal dimension $s=2$ of the state space keeps the discussion of dynamic properties simple and, furthermore, allows for parallels to previous dynamic models and work on elementary CAs [14].

Different attempts to classify the rule space of CAs have been carried out. Wolfram [15] divided CAs qualitatively into four classes, according to the emerging spatiotemporal patterns and analogous to dynamical systems descriptions: I (homogeneous stationary 
state), II (heterogeneous stationary state or simple periodic structures), III (chaotic behavior), and IV (long range correlations and propagating structures). The introduction of the Langton parameter $\lambda$ [16] allowed a quantitative investigation of CA rules, even for large rule spaces. While for $s>2$ Langton's scheme of generating a transition function for a given value of $\lambda$ requires some statistical subsidiary conditions, for binary CAs $\lambda$ is simply the number of neighborhoods mapped onto the state 1 divided by the number of all possible neighborhood states. For rules generated with the "random-table method", where $\lambda$ is used as the probability for a neighborhood to be mapped on state 1, the Langton parameter defines trajectories through the $\mathrm{CA}$ rule space and, consequently, through the four different dynamical regimes. The order, in which the corresponding Wolfram classes are passed as $\lambda$ is increased, is I $\rightarrow$ II $\rightarrow$ IV $\rightarrow$ III. Class IV automata, lying between periodic (II) and chaotic (III) behavior and exhibiting long-range correlations and propagating structures, are regarded as suitable scenarios for the study of complexity and self-organization. A recent, alternative parametric approach is given in [17]. There, the authors introduce a parameter $F$ controlling details of the transition function beyond the $\lambda$ parameter and show the existence of all Wolfram classes for a given $\lambda$ and an appropriate $F$. Obviously, the diversity of classes in a subset of rules with a given $\lambda$ depends on the way the rules are generated.

Our paper is structured as follows: In Section 2, we formulate two classes of binary cellular automata on graphs and discuss the resulting dynamics. While the first class keeps the Langton parameter $\lambda$ constant, $\lambda$ varies with the parameter $\kappa$ for the second class. In the Section 3, we focus on complex spatiotemporal patterns as an indicator of optimal information transport and introduce statistical tools for segregating different dynamic domains. Applied to ordinary CAs, the Shannon entropy $S$ and the word entropy $W$ allow an adequate qualitative separation of the four Wolfram classes. Similarly to Langton's investigation, the pattern formation capacity of cellular automata can thus be quantitatively analyzed in a two-dimensional plane spanned by the two entropy measures. In Section 4 we continuously change topological parameters of the conventional ring lattice and implement the two sets of binary dynamics. A link between CAs on graphs, as described in this investigation, and more traditional forms of CA studies is provided by analyzing regular graph topologies with different neighborhood sizes. We then study procedures of topology variations and compare the emerging patterns of small-world and random graphs with the well-classified ones from CAs in terms of entropy measures. Finally, we study the pattern formation capacity of scale-free graphs and randomized variants with the same degree distribution. Section 5 gives a mean-field analysis of the dynamic behavior of random graphs based on the local state densities which govern the nodes' dynamics. In this way we gain insight into the mechanisms of the considered dynamics and the link between some topological and dynamical features. The last section (Section 6) reviews the results, discusses implications for the dynamics on real networks and lines out some ideas for further investigations. 


\section{Cellular automata on graphs}

The topology of a 1D CA is usually a regular ring lattice with a clustered neighborhood structure, i.e. a graph of $N$ nodes forming a closed chain with additional edges linking each element to a certain number of neighbors. In such a graph each node has the same degree $k$ due to the links to the $k / 2$ neighbors in both directions along the chain. In this paper we will refer to this specific ring lattice topology as a regular graph. Note, however, that in graph theory this term is used for all networks with a delta-like degree distribution. Since in CAs the transition function $\Delta$ is defined for a fixed number of neighbors $k$, this system does not provide an appropriate framework for studying changes in the system's architecture. A change of $k$ would correspond to an alteration of the underlying rule space, which restricts comparability of two systems with different degree. We therefore introduce two sets of functions $\Omega_{1}(\kappa)$ and $\Omega_{2}(\kappa)$, each depending on a single parameter $\kappa$, which account for the individually varying architecture and, consequently, allow the formulation of binary CAs on arbitrary graphs.

Let the graph be represented by its adjacency matrix $A_{i j}$. When a link exists between nodes $i$ and $j$ of the graph, the corresponding matrix element is 1 , and it is 0 otherwise. We consider only undirected graphs. Consequently, the adjacency matrix is symmetric, $A_{i j}=A_{\ddot{j}}$. We call the first set of rules $\Omega_{1}$. There, the state $x_{i}$ of node $i$ of a graph flips its state in the next time step if the density of 1 's among the $k_{i}$ elements linked to node $i, \rho_{i}$, is larger than a parameter $\kappa$,

$$
\Omega_{1}(\kappa): x_{i}(t+1)= \begin{cases}x_{i}(t), & \rho_{i} \leq \kappa \\ 1-x_{i}(t), & \rho_{i}>\kappa\end{cases}
$$

There, $\rho_{i}$ is defined by

$$
\rho_{i}=\frac{1}{k_{i}} \sum_{j} A_{i j} x_{j}(t) .
$$

We will refer to this quantity as the local density. Since the sum does not include $x_{i}$ itself, the action on node $i$ is invariant under the changing of the central element. This symmetry is broken by introducing

$$
\Omega_{2}(\kappa): x_{i}(t+1)= \begin{cases}x_{i}(t), & \tilde{\rho}_{i} \leq \kappa \\ 1-x_{i}(t), & \tilde{\rho}_{i}>\kappa\end{cases}
$$

where now the density $\tilde{\rho}_{i}$ incorporates the state $x_{i}$ itself:

$$
\tilde{\rho}_{i}=\frac{1}{k_{i}+1}\left(x_{i}(t)+\sum_{j} A_{i j} x_{j}(t)\right) .
$$


In $\Omega_{1}$ the state $x_{i}(t+1)$ depends on the sum over the neighboring states and on the state $x_{i}(t)$ itself,

$$
\Omega_{1}: x_{i}(t+1)=f\left(x_{i}(t), \sum_{j} A_{i j} x_{j}(t)\right),
$$

whereas in $\Omega_{2}$ the state $x_{i}(t)$ is also included in the sum. Notably, all rules defined by $\Omega_{i}$ are legal, according to Wolfram's definition [14], i.e. they fulfill the quiescent condition (a configuration of only zeroes is unchanged) and reflection symmetry (001 and 100 are mapped onto the same state).

In order to get familiar with the set of functions $\Omega_{1}$ and $\Omega_{2}$, it is instructive to apply the rules to regular graphs first. In this case, each $\Omega_{i}(\kappa)$ is identical to a corresponding CA rule $\Delta$. Table 1 shows the neighborhood mappings for an elementary CA, i.e., a CA with $s=2$ and $k=2$. By inspecting the rule table, we can easily infer the corresponding CA rules: $\Omega_{1}\left(0 \leq \kappa<\frac{1}{2}\right) \equiv$ rule $00110110_{2} \equiv$ rule 54 etc. Earlier investigations on elementary CA [14] showed that these rules correspond to stationary (rules 36, 76, 108 and 204), oscillatory (rule 50) and complex (rule 54) behavior, which can also be inferred in a natural way from the definitions (11) and (3): According to (1), for small $\kappa$ most nodes will change their state and an oscillatory behavior will dominate, while for large $\kappa$ the majority of nodes will not change their state and a stationary pattern will prevail. In between these limiting regimes we expect complex and chaotic behavior.

[Table 1 about here.]

As can be inferred from Tab.11 the Langton parameter of all $\Omega_{1}$ rules is constant, $\lambda=0.5$, since the number of neighborhoods mapped on 0 and 1 is equal. This is a generic property of $\Omega_{1}$. Let $\boldsymbol{y}_{i}=\left\{x_{i-k / 2}, \ldots, x_{i+k / 2}\right\}$ denote the neighborhood configuration of node $i$ excluding the state $x_{i}$ itself. Then, under $\Omega_{1}(\kappa)$ a neighborhood configuration $\boldsymbol{y}$ will flip a central 0 as well as a central 1 for an appropriate value of $\kappa$. Since the identical mapping (rule 204) is characterized by $\lambda=0.5$ and the number of flipping 0's and 1's is equal, $\lambda=0.5$ for all $\Omega_{1}$ rules. Therefore, this set of rules exploits a pattern formation capacity orthogonal to Langton's investigation and, as we will see, proves the existence of all dynamic domains for $\lambda=0.5$. This is an elementary example of changing the Wolfram class at constant $\lambda$ as stressed in [17].

The lack of the invariance with respect to the central element in $\Omega_{2}$ is responsible for a varying Langton parameter in this case. We find that the number of 1 's in the rule table (and accordingly $\lambda$ ) varies with $\kappa$, as shown in Tab.11 In the regime where oscillatory and stationary behaviors coexist and complex behavior can be expected (i.e. $\frac{1}{3} \leq \kappa<\frac{2}{3}$ ) most neighborhoods are mapped on 0 and the system is prone to a density loss in the course of time. 


\section{Quantitative classification of spatiotemporal patterns}

In this section we present two entropy measures and apply them to conventional CAs in order to separate and categorize general spatiotemporal patterns of such CAs. By selecting only quantities evaluating the time development of single elements (as opposed to whole spatiotemporal configurations) this classification scheme can, after gauging it with conventional CAs, be directly applied to CAs on graphs, where the degree $k$ is given by a distribution $P(k)$.

The Shannon entropy $S$ relies on the probability for the emergence of the cell states $x \in \Sigma$ in the time evolution of cell $i$,

$$
S_{i}=-\sum_{j=0}^{s-1} p\left(x_{j}\right) \log _{2} p\left(x_{j}\right) .
$$

For binary dynamics, the $p\left(x_{j}\right)$ are the probabilities for 0's and 1's. Averaging $S_{i}$ over all $N$ cells yields the average Shannon entropy $S=\sum_{i=1}^{N} S_{i} / N$. All stationary patterns result in $S=0$, while random and oscillatory patterns yield $S=1$ because of the equal distribution of 0's and 1's in the time series of each cell.

The word entropy $W$ uses the occurrence of blocks of constant cells of length $l$ ( $l$-words) in the time series of cell $i$,

$$
W_{i}=-\sum_{l=1}^{t} p(l) \log _{2} p(l),
$$

where $p(l)$ is the probability for an $l$-word, irrespective of the state $x$ this word consists of. The maximal word length is clearly the length of the time series $T$. The average value of the word entropy is given by $W=\sum_{i=1}^{N} W_{i} / N$. The word entropy is similar to Wolfram's "measure entropy" [15], but instead of spatial blocks we use temporal correlations here. Moreover, $W$ measures solely the occurrence of blocks of constant cells and not all possible blocks of length $l$.

Both entropy measures analyze the temporal behavior of cells. The drawback of this definition is clearly the dependence of $W$ on the length $T$ of the time series of the pattern. However, this problem exists for the spatial variant (i.e. the measure entropy), when finite-size CAs are considered. As pointed out above, the advantage of the measures presented here is their generic applicability to arbitrary topologies, where the analyzed spatiotemporal patterns lose the spatial information because of the irregular neighborhood configurations.

Alternatives for the word entropy are given by, e.g., the mutual information and by spectral properties of average-density fluctuations. The Shannon entropy on the other hand exhibits partially similar properties as the Hamming distance. We checked that all these quantities lead to analog qualitative results. 
With these tools available we can visualize the separation of CAs within a WS plane. Rather than inferring rule space properties, we want to locate domains of spatiotemporal patterns on this plane. We therefore gauge the plane with $k=10 \mathrm{CAs}$, where the initial cell states 0 and 1 have been randomly assigned. We generate the different CA rules according to Langton's "random-table method", but other parameterizations like the one proposed in [17] could be used as well. Fig. 1] shows typical patterns of $N=500, k=10$ CAs. We can now classify the patterns according to their $W$ and $S$ values within the $W S$ plane. Stationary patterns lie in the lower left corner of the plane, while purely oscillatory ones are located in the lower right. Chaotic patterns are localized in a rather small region in the upper right part of the plane. Between these extremal regions, the plane is filled with CAs with partly non-trivial periodic structures and patterns with long-range correlations.

[Figure 1 about here.]

We can now plot the $\Omega_{1}(\kappa)$ and $\Omega_{2}(\kappa)$ rules in the $W S$ plane to visually infer the effect of increasing $\kappa$. All the following simulations have been carried out on graphs with 500 nodes and random initial condition, i.e., every cell is independently assigned a value $x_{i} \in$ $\{0,1\}$ at time $t=0$, resulting in an initial density $\rho(t=0)$ of about 0.5 . We always drop the first 500 time steps and use the second 500 steps to calculate the entropy measures. We display the medians of five runs in all following figures to ensure statistical reliability. In Fig. 2 we show the gauged plane with the four Wolfram classes qualitatively assigned to specific regions. This allows the immediate identification of the different dynamic domains as $\kappa$ is increased for $\Omega_{1}(\kappa)$ on a regular graph with 2 neighbors or, in the language of CAs, the succession of rules 54, 108, 204 for $k=2$ CAs. Three snapshots show the corresponding patterns, where we selected 100 nodes and 100 time steps for visual clarity.

[Figure 2 about here.]

A larger number of rules with Langton parameter $\lambda=0.5$ can be studied for a larger neighborhood size. Fig. 3 shows the trajectory of $k=10$ CAs for increasing $\kappa$ in the gauged $W S$ plane. In this case, the transition from oscillatory to stationary behavior is accompanied by complex structures in the region of class IV automata. Large $W$ values emerge for every $\kappa \in[0.3,0.4$ [, where the exact parameter value is irrelevant because of the discrete number of different local densities.

[Figure 3 about here.]

Fig. 4 shows $\Omega_{2}(\kappa)$ for $k=2$ CAs. In the region $\frac{1}{3} \leq \kappa<\frac{2}{3}$, where one would expect complex patterns analogous to $\Omega_{1}$, only two neighborhood configurations are mapped onto the state 1 (see Tab. 1), resulting in a density loss and a stationary pattern where 0's prevail (Fig. $4 \mathrm{p})$.

[Figure 4 about here.] 


\section{Topology variation}

For $\Omega_{1}$ the variation of $\kappa$ for regular graphs explores the rule space orthogonal to the Langton parameter. Different neighborhood sizes result in different trajectories within the WS plane. We find the Wolfram classes II,IV and I as $\kappa$ is increased. This transition from oscillatory to stationary behavior is a generic property of the sets $\Omega_{i}$.

However, in this chapter we want to focus on another feature of the two rule sets, namely their applicability to arbitrary topologies. Regardless of the global network structure, single nodes evolve according to their local density in every time step. We can monitor the dynamical changes of a network along the topological variations. To do so, we first have to select an adequate $\kappa$ value where the capacity of producing complex patterns is high, as we expect that in this configuration the patterns are most sensitive to the topology of the underlying graph. Fig. 5 shows the word entropy vs. $\kappa$ for $k=6$ and $k=10$ regular graphs and, since we will deal with random graphs later on, for a random graph of the Erdős and Rényi type [18] with a mean degree of 10.

[Figure 5 about here.]

In order to encounter a large variety of patterns and high word entropies for both regular and random graphs, we choose a $\kappa$ value within the interval $[0.35,0.4[$. In the following simulations, $\kappa=0.36$ will be used as the parameter value for the $\Omega_{i}$, but similar values of $\kappa$ would serve as well, as can be inferred from Fig. 5

As a first step we continue analyzing regular graphs - and thereby allow for conventional CA interpretation - but increase the neighborhood size $k$ for every node in even numbers. In this case, $\Omega_{i}(0.36)$ corresponds to a different CA transition rule $\Delta$ for each neighborhood size $k$. Fig. 6 shows $W$ and $S$ of spatiotemporal patterns of $\Omega_{1}$ and $\Omega_{2}$ (top) on regular graphs against increasing neighborhood size $k$. The small difference in the definition of the two sets of rules leads to striking differences of the dynamic response for regular graphs. For small $k$ we find a multiple peak structure in the entropy measures for $\Omega_{1}$ and a variety of different spatiotemporal patterns. For $\Omega_{2}$, the word entropy $W$ is close to 0 for all neighborhood sizes. For large neighborhoods around $k>30$, the dynamics is purely oscillatory, indicated by $S=1$ and $W=0$. The lower picture in Fig. 5 shows the path for $\Omega_{1}$ in the $W S$ plane as $k$ is increased. The peak structure in the picture above appears now as jumps between different Wolfram classes.

[Figure 6 about here.]

The entropy values for large $k$ can be understood in the limiting case of a completely connected graph (complete graph) which corresponds to CAs with $k=N-1$. There, the neighborhood is approximately identical for all nodes and the overall dynamics is governed by the initial density $\rho(t=0)$. For $\rho(0) \leq \kappa$ all nodes remain in their initial 
state, for $\rho(0) \wedge 1-\rho(0)>\kappa$ the nodes oscillate constantly, while for $1-\rho(0) \leq \kappa<\rho(0)$, all nodes flip their state in the first time step and then remain constant. Above a certain critical value of the connectivity, all elements have essentially the same neighborhood and consequently display the same dynamical behavior. This threshold is independent of other topological details and corresponds to the synchronization threshold known from (continuous) dynamical systems.

If we decrease $k$, the densities of 1's in the neighborhoods $\boldsymbol{y}_{i}=\left\{x_{-k / 2}, \ldots, x_{k / 2}\right\}$ increasingly differ and at some point, these differences lead to a coexistence of stationary and oscillatory behavior, i.e., complex system dynamics can emerge. If we regard $\Omega_{2}$, we find that the coexistence of stationary and oscillatory behavior is not sufficient for the emergence of complex system dynamics. There, the transition between the stationary and oscillatory domains is accompanied by spatially clearly separated blocks of different behavior, as can be seen in the pattern examples in Fig. 6.

A second way to study the influence of topology is to gradually rewire the original regular graph. We can conduct this procedure with the preservation of the degree distribution $P(k)$ (randomization) or without preserving $P(k)$ (rewiring), resulting in two different topologies. If the degree distribution $P\left(k^{\prime}\right)=\delta_{k, k^{\prime}}$ is conserved, we end up with a regular graph where the $k$ couplings of every node are randomly chosen out of the $N-1$ remaining nodes. We will refer to this type of graph as the DDR (delta-distributed random) graph. Note that DDR graphs can also be considered as undirected Kauffman networks [19]. In the other case, where the degree distribution is altered in the course of the rewiring, we end up with a binomial degree distribution and a mean degree $k$. This limiting case coincides with the ER graph discussed above. Such a rewiring procedure has first been introduced by Watts and Strogatz [6] as a model of small-world graphs. Fig. 7 visualizes the two methods for a regular ring lattice with $N=15, k=4$.

[Figure 7 about here.]

In both cases, the strict analogy to a $\mathrm{CA}$ and a concrete rule numbering is lost as soon as the architecture differs from a regular nearest-neighbor configuration and the applicability of the $\Omega_{i}$ to arbitrary topologies is exploited. In Fig. 8 we display the emerging spatiotemporal patterns as the rewiring and randomization depth $p$ is increased. This quantity is the ratio of rewired links to all existing links. While for $\Omega_{1}(\kappa=0.36)$, both procedures result in similar patterns, $\Omega_{2}$ resolves the different degree distributions: the two architectures (ER and DDR ) lead to stationary and non-trivial patterns respectively. However, we have to note that the linear arrangement of nodes according to the node number in the spacetime plots has no topological foundation. Adjacent nodes in the pattern do no longer have to be linked to each other as the underlying graph is rewired.

[Figure 8 about here.] 
Fig. 9 visualizes the trajectories of the two procedures for $\Omega_{1}$ and $\Omega_{2}$ in the gauged $W S$ plane and locates the corresponding pattern formation capacity. The decrease of the word entropy for $\Omega_{1}$ relies on the continuous disintegration of clustered neighborhoods. For $\Omega_{2}$ we find the opposite behavior: The clustering inhibits complex patterns for this parameter setting and only the randomization of links allows for non-trivial structures. The large coverage of dynamic domains demonstrates the large and systematic regulation of pattern formation capacity by topology.

[Figure 9 about here.]

We can close the link between the two topological variations by increasing stepwise the degree $k$ in the generated networks, ending up with complete graphs, as discussed in the beginning of this section. In Fig. 10 we show the procedures for small ring lattices for ER and DDR graphs respectively.

[Figure 10 about here.]

Fig. 11] shows the effect of increasing $k$ on the word entropy $W$ of patterns of ER and DDR graphs for both $\Omega_{i}$. The increase of the neighborhood size results in the leveling of individual neighborhood density differences. For $k>60$ all nodes see approximately the same density of 1's within the linked elements and follow a collective behavior (oscillation for this $\kappa$ ). In contrary to the regular graphs, ER and DDR graphs are both capable of generating complex patterns for $\Omega_{2}$.

[Figure 11 about here.]

The class of graphs, which received particular scientific attention in the last few years, are scale-free graphs [7] whose power-law degree distribution is ubiquitous in nature. Therefore, it is interesting to assess, how topological variations affect the dynamic behavior and moreover, if these graphs show some kind of extremal pattern formation capacity.

We use the Barabási-Albert (BA) algorithm [7] to generate graphs and then apply three algorithms, which change the topology but keep the degree distribution $P(k)$ constant as described in [20]. We randomize, hierarchize and antihierarchize the networks by rewiring pairs of edges according to the corresponding reconnection rule. Randomization rewires two pairs of linked nodes randomly. The hierarchization process connects nodes with similar degree and thus imposes an ordering of degrees within the network. This results in chains of linked cells with increasing degree and interconnected hubs, i.e., the nodes with extremely high degree are linked together. Antihierarchization, though, links nodes with high and low degree and levels hierarchical structures in the network. The degree correlations of graphs generated with the latter two procedures resemble the assortative and disassortative mixing observed in real networks [21]. Fig. 12] shows the effect of randomizing a BA scale-free graph with minimal degree $k_{0}=4$ up to a randomization depth of $p=2.5$ within a section of the $W S$ plane for $\Omega_{1}(0.36)$. Each process affects the 
response of the system. While randomization increases and antihierarchization decreases $W$ slightly at nearly constant $S$, the hierarchization process yields maximal $W$ values for a randomization depth of $p \approx 2$ and a relatively large variation of $S$.

The extremely inhomogeneous degree distribution of this kind of graphs results in an inhomogeneous word entropy distribution, contrary to regular graphs, where the nodes are indistinguishable with respect to their degree, and the distribution is delta-like. The inset in Fig. 12] shows $W_{i}$ against the degree $k_{i}$ for all nodes of a BA scale-free graph. Obviously, the hubs in the system account for small $W$, while nodes with a minimal degree are responsible for large and maximal $W$. Concerning the pattern formation capacity of hierarchize scale-free graphs, the maximal $W_{i}$ generated by single nodes increases with the degree of hierarchization up to a saturation level. This level around $W=4$ for 2000 hierarchization steps lies clearly above the maximal values observed for conventional CAs.

[Figure 12 about here.]

\section{Density distribution analysis}

In the previous section we found that a large number of neighbors synchronizes the system and thus inhibits complex behavior. In this section, we want to put the argument on a more quantitative level, infer the domain of dynamic behavior from the distribution of the individual densities $P\left(\rho_{i}\right)$ and give an explanation for the characteristic structure of the word entropy as $k$ is increased for random graphs (Fig.111).

The local densities $\rho_{i}(t)$ and $\tilde{\rho}_{i}(t)$ defined in Eqs. (2) and (4) control the evolution of node $i$ at time $t$ for both rule sets. The dynamics therefore depend on the parameter $\kappa$ and on the topology of the underlying graph, which defines the numbers and positions of the linked elements. Let us consider the case of an initial state density $\rho(0)=0.5$. The topology then determines the density distribution $P\left(\rho_{i}\right)$. In all the following discussions, we assume that $P\left(\rho_{i}\right)$ is time independent and neglect deformations of $P\left(\rho_{i}\right)$ in the course of time. In this case, essential features of the dynamics can be immediately derived.

[Figure 13 about here.]

In the discussion of Fig. 6 we argued that for large $k$ all nodes react according to the same density $\rho_{i} \approx \rho(0)$ and are therefore synchronized. For smaller $k$ values, the differences in the individual densities $\rho_{i}$ account for the coexistence of dynamic behaviors. We can visualize this difference by plotting the distribution of individual densities, $P\left(\rho_{i}\right)$, for a small and a large neighborhood, together with the parameter $\kappa$ in Fig. 13, Cells with densities left of the $\kappa$ line are stationary, while cells to the right change their state. For a large neighborhood with $k=50$, nearly all nodes oscillate according to their large local densities $\rho_{i}>\kappa$. However, for $k=10$, we find a considerable fraction of cells stationary and argue that this coexistence of dynamic domains allows for complex behavior. Note 
that the distributions in Fig. 13 are not normalized but scaled to the identical maximum value. This is done because only the ratio of oscillatory and stationary cells at fixed $k$ provides the relevant information on the dynamics.

The peak structure of the word entropy for graphs with increasing neighborhood size $k$ in Figs. [ and 11 is evident for both regular and DDR graphs. We can explain this structure for DDR graphs with the following mean-field approach for $\Omega_{1}$. Let $\boldsymbol{y}_{i}$ be the configuration of the coupled states of node $i, \boldsymbol{y}_{i}=\left\{x_{i 1}, \ldots, x_{i k}\right\}$. Node $i$ remains constant, if its local density $\rho_{i}$ is smaller than $\kappa$. Thus, the fraction $r$ of constant cells is the sum over all neighborhood configuration with $\rho \leq \kappa$,

$$
r=\sum_{m=0}^{\lfloor(\kappa k)} p(m, \rho(0))=\sum_{m=0}^{\lfloor(\kappa k)}\left(\begin{array}{c}
k \\
m
\end{array}\right) \rho(0)^{m}(1-\rho(0))^{k-m},
$$

where $\left\lfloor(z)\right.$ is the greatest integer less or equal $z, m$ the number of 1 's within $\boldsymbol{y}_{i}$ and $p(m)$ is the probability for finding $m$ out of $k$ neighbors in state 1 for the initial density $\rho(0)$ and $\Omega_{1}$. In Fig. 14 we plot this number $r$ against the neighborhood size with parameters $\kappa=0.36$ and an initial density of $\rho(0)=0.5$ and compare it to the $W$ values observed in a numerical simulation. The simulated curve is identical to the one in Fig. 11, $\Omega_{1}$, apart from the now enlarged degree range from 2 to 80 .

[Figure 14 about here.]

The direct proportionality between $r$ and $W$ over a wide range of $k$ supports the approach to link the initial settings with the corresponding dynamic domain. However, a discrepancy for $k=4$ and $k=6$ is obvious, since the word entropy values are 0 for this degrees. We argue, that $r$ values above 0.3 inhibit complex behavior in this DDR graph because of too many stationary elements. This threshold has to be inferred from the comparison of the simulated $W$ and the calculated $r$ values. Nevertheless, together with this threshold, we can predict the complexity of the time evolution simply from the topology and the initial local densities for random graphs. This fails for regular graphs, as can be seen from Fig. 6, where the shape of $W$ differs clearly from the one in Fig. 14 For those graphs, the density distribution $P\left(\rho_{i}\right)$ is well responsible for the peak structure and identical at $t=0$ but the spatial correlations emerging in this topology because of the clustered neighborhoods alter this distribution in the course of time.

We can generalize the mean-field approach to other topologies by incorporating the degree distribution $P(k)$ in Eq. 8 ,

$$
r(k, \kappa, \rho(0))=\sum_{k=0}^{N-1} P(k) \sum_{m=0}^{\left\lfloor\left(\kappa k^{\prime}\right)\right.} p(m, \rho(0))
$$

In this way we can explain the smoothing of the peak structure for $E R$ graphs as $k$ is increased in Fig. 11. Moreover, Eq. (9) can be applied to infer the dynamic behavior of 
scale-free graphs. Instead of varying the degree $k$, we now study the dynamic behavior as $\kappa$ is increased from 0 to 0.5 . Fig. 14b shows $W(\kappa)$ from a simulation and the calculated $r$ for a BA scale-free graphs with minimum degree $k_{0}=5$. Again, the qualitative trend and the jumps in the word complexity are well predicted by $r$. For $r>0.3$ the complex behavior collapses and $W$ drops sharply to 0 . Thus, BA scale-free graphs can be described properly by the mean-field approach for random graphs, i.e. their local density distribution remains approximately constant. Notably, with a slight alteration of $p(m, \rho(0))$, the set of rules $\Omega_{2}$ can be also described by Eq. (9).

\section{Conclusions}

The role of topology for dynamics is becoming one of the key topics of nonlinear dynamics and the theory of self-organization. In this paper we show by numerical simulation that the pattern formation capacity of binary CAs strongly depends on the topology of the underlying graph. We used two temporal entropies, the Shannon entropy $S$ and the word entropy $W$, to separate the different dynamic domains. We formulated two classes of binary cellular automata on graphs, $\Omega_{1}$ and $\Omega_{2}$, each depending on a single parameter $\kappa$. While the first class keeps the Langton parameter $\lambda$ constant, $\lambda$ varies with $\kappa$ for the second class. If applied to regular graphs, the two sets $\Omega_{i}$ naturally parameterize and categorize a subset of CA rules. Thus, we studied the transition between stationary and oscillatory behavior for these two CA rule sets. We assessed the influence of the neighborhood size $k$ for the pattern formation capacity of CAs for a rule that accounts for increasing $k$. For large $k$, we observed synchronization of the elements and the absence of complex patterns. Beyond the conventional CA topology, we investigated the effect of topological variations. We found a crucial dependence on the central element by comparing the two rule sets. We found that a continuous change in the topological parameters of a graph can lead to a continuous trajectory in the $W S$ plane. Moreover, we studied the pattern formation capacity of modified BA scale-free graphs. Hierarchizing such a network leads to a increase in the word entropy for low-degree nodes.

The following observations on the link between topology and dynamics have been made:

- The inclusion of the state of cell $i$ itself into the local density has a fundamental impact on the emerging dynamics. For all graph types we found distinct differences between the two sets $\Omega_{1}(\kappa)$ and $\Omega_{2}(\kappa)$ for a large range of the parameter $\kappa$.

- Graphs with a delta-like degree distribution display a characteristic peak structure in the word entropy as the neighborhood size is increased from small values (cf. Fig. 6 and Fig. 11 for regular and DDR graphs respectively). This crucial dependence on the number of neighbors allows for multiple transitions between different dynamic domains as $k$ grows. This is due to the discrete distribution of local densities and 
can be understood with a mean-field approach. However, the local correlations in the CA topology account for the enormous jumps in this case.

- Rewiring a regular topology disintegrates the clustered neighborhoods and destroys gradually local collective behavior. Beyond the small-world regime, the ability to produce long-range correlations is lost. If differences of the rewiring process (conservation or alteration of the degree distribution) can be resolved or not depends decisively on the definition of the dynamics applied (cf. Fig. 9).

- In scale-free graphs a variation of the degree correlations changes the word entropy of the time series of individual nodes. The maximal $W_{i}$ values occur for nodes in the low-degree domains of hierarchized topologies and lie far above their regular counterparts. However, there are also nodes with small $W_{i}$ for all degrees, resulting in an average $W$ within the range of values from conventional CAs.

Aside from trajectories of topological variations, the path of a graph along the variation of $\kappa$ may be used for the topological characterization. In principle, the trajectory in the $W S$ plane as a function of $\kappa$ (e.g., for $\Omega_{1}$ ) could serve as a dynamical signature of a particular graph, which assesses the graph's capacity to display complex dynamics. We found that the two rule sets are sensitive to variations in different network types. A detailed study of the connection between the dynamic response and such graph differences, even up to the motif level, along $\kappa$, may be an adequate prerequisite for the characterization of real biological and technical graphs with the means of the rule sets proposed in this paper. Eventually, it would be interesting to implement our CA rules, e.g., on protein interaction graphs and compare this dynamical signature with those from the graph's randomized counterparts in search for an evolutionary optimization on this level.

\section{References}

[1] H. Jeong, B. Tombor, R. Albert, Z. N. Oltvai, and A.-L. Barabási. The large-scale organization of metabolic networks. Nature 407, 651 (2000).

[2] D. A. Fell and A. Wagner. The small world of metabolism. Nature Biotechnology 18, 1121 (2000).

[3] M. E. J. Newman. The structure of scientific collaboration networks. PNAS 98, 404 (2001).

[4] H. Jeong, S. P. Mason, A.-L. Barabási, and Z. N. Oltvai. Lethality and centrality in protein networks. Nature 411, 41 (2001). 
[5] I. J. Farkas, I. Derényi, H. Jeong, Z. Neda, Z. N. Oltvai, E. Ravasz, A. Schurbert, and A.-L. Barabási. The topology of the transcription regulatory network in the yeast, Saccharomyces cerevisiae. Physica A 381, 601 (2003).

[6] D. J. Watts and S. H. Strogatz. Collective dynamics of 'small-world' networks. Nature 393, 440 (1998).

[7] A.-L. Barabási and R. Albert. Emergence of scaling in random networks. Science 286, 509 (1999).

[8] S. H. Strogatz. Exploring complex networks. Nature 410, 268 (2001).

[9] A. Barrat and M. Weigt. On the properties of small-world network models. European Physical Journal B 13, 547 (2000).

[10] M. Aldana. Boolean dynamics of networks with scale-free topology. Physica D 185, 45 (2003).

[11] R. Pastor-Satorras and A. Vespignani. Epidemic spreading in scale-free networks. Phys. Rev. Lett. 86, 3200 (2001).

[12] M. Barthélemy, A. Barrat, R. Pastor-Satorras, and A. Vespignani. Dynamical patterns of epidemic outbreaks in complex heterogeneous networks. condmat/0410330, (2004).

[13] S.-Y. Huang, X.-W. Zou, Z.-J. Tan, and Z.-Z. Jin. Network-induced nonequilibrium phase transition in the "game of Life". Phys. Rev. E 67, 026107 (2003).

[14] S. Wolfram. Statistical mechanics of cellular automata. Rev. Mod. Phys. 55, 601 (1983).

[15] S. Wolfram. Universality and complexity in cellular automata. Physica D 10, 1 (1984).

[16] C. G. Langton. Computation at the edge of chaos: phase transitions and emergent computation. Physica D 42, 12 (1990).

[17] S. Sakai, M. Kanno, and Y. Saito. Quiescent string dominance parameter F and classification of one-dimensional cellular automata. Phys. Rev. E 69, 066117 (2004).

[18] P. Erdős and A. Rényi. On random graphs. Publicationes Mathematicae 6, 290 (1959).

[19] S. A. Kauffman. Metabolic stability and epigenesis in randomly constructed nets. Journal of Theoretical Biology 22, 437 (1969). 
[20] A. Trusina, S. Maslov, P. Minnhaben, and K. Sneppen. Hierarchy measures in complex networks. Phys. Rev. Lett. 92, 178702 (2004).

[21] M. E. J. Newman. Assortative mixing in networks. Phys. Rev. Lett. 89, 208701 (2002). 


\section{List of Figures}

$1 \quad$ Typical patterns of the different dynamic domains within the $W S$ plane. The plane is filled with $N$ 2 Behavior of $\Omega_{1}$ for elementary, i.e. $k=s=2$, CAs within the $W S$ plane as $\kappa$ is increased. The thr 3 Behavior of $\Omega_{1}$ for $k=10$ CAs within the $W S$ plane as $\kappa$ is increased. Only for $\kappa \in[0.3,0.4[$ (b) c $4 \quad$ Behavior of $\Omega_{2}$ for elementary CAs within the $W S$ plane as $\kappa$ is increased. The corresponding rule 5 The word entropv $W$ against the variation of $\kappa$ for $\Omega_{1}$ for two regular graphs and an $E R$ random $g$ $6 \quad$ Word entropv (solid) and Shannon entropv (dashed) as a function of neighborhood size $k$ for $\Omega_{1}$ an $7 \quad$ Rewiring (top) and randomization (bottom) of a regular graph. In the first case, the resulting degre $8 \quad$ Spatiotemporal patterns for the different rewiring mechanisms described above for increasing rewi $9 \quad$ The four traiectories for $\Omega_{1}$ and $\Omega_{2}$ for the rewiring and randomization process of an originally reg 10 Increase of the degree for ER and DDR graphs. We show graphs with 15 nodes for $k=4.8 .12 .14$ 11 Word entropv $W$ for increasing $k$ for DDR (solid) and ER (dotted) graphs and both rule sets. DDR 12 Effects of randomization (dashed), hierarchization (grav) and antihierarchization (grav) of a BA sc 13 Scaled density distributions $P\left(\rho_{i}\right)$ for DDR graphs with $k=10$ and $k=50$. The line defined by $\kappa$ 14 Fraction of constant cells $r$ compared with the word entropy $W$ for two random topologies for $\Omega_{1}$. 

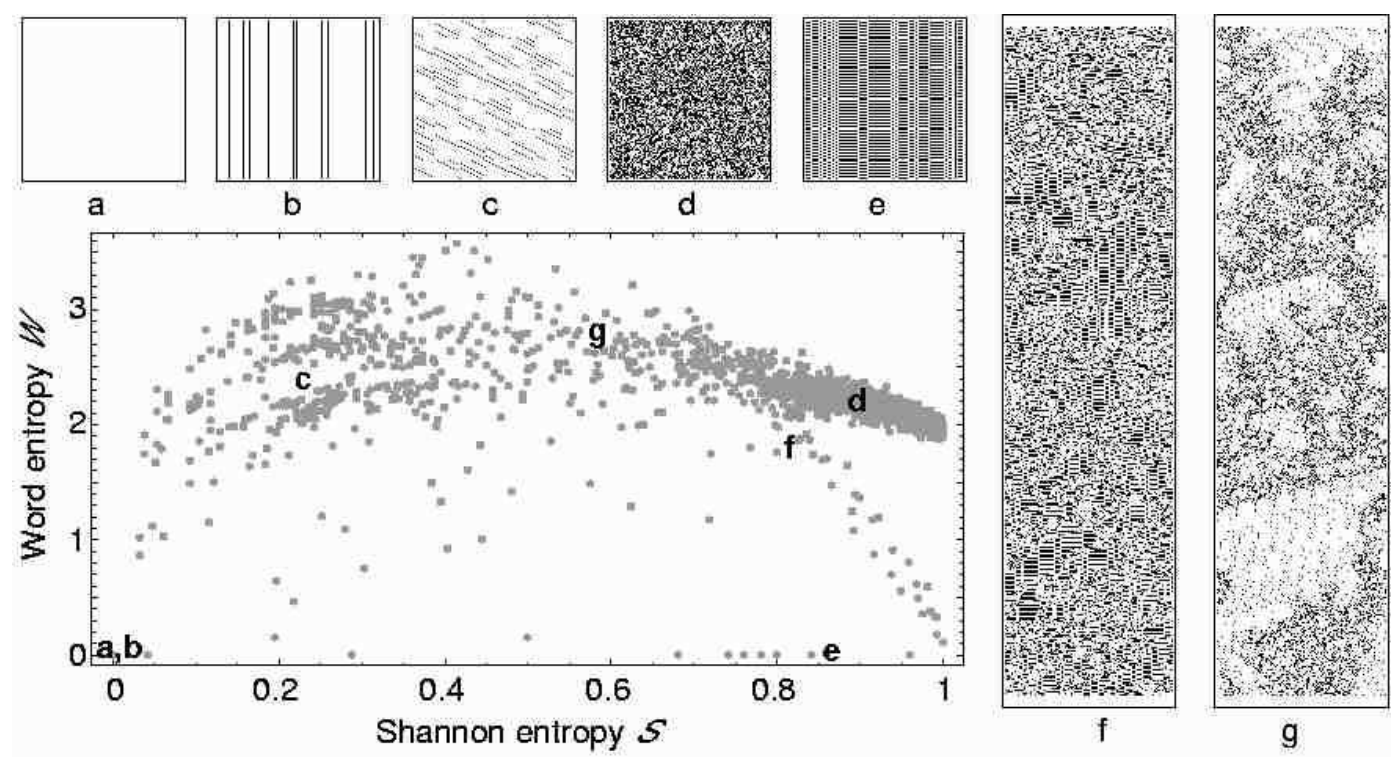

Figure 1: Typical patterns of the different dynamic domains within the $W S$ plane. The plane is filled with $N=500, k=10$ CAs where the time interval $] 500,1000]$ has been used to calculate $W$ and $S$. Patterns of stationary, oscillatory, periodic and chaotic automata, (a) - (f), comprise 500 time steps, the two class IV patterns (f) and (g) comprise 2000 time steps. 0's are indicated black, 1's white. 


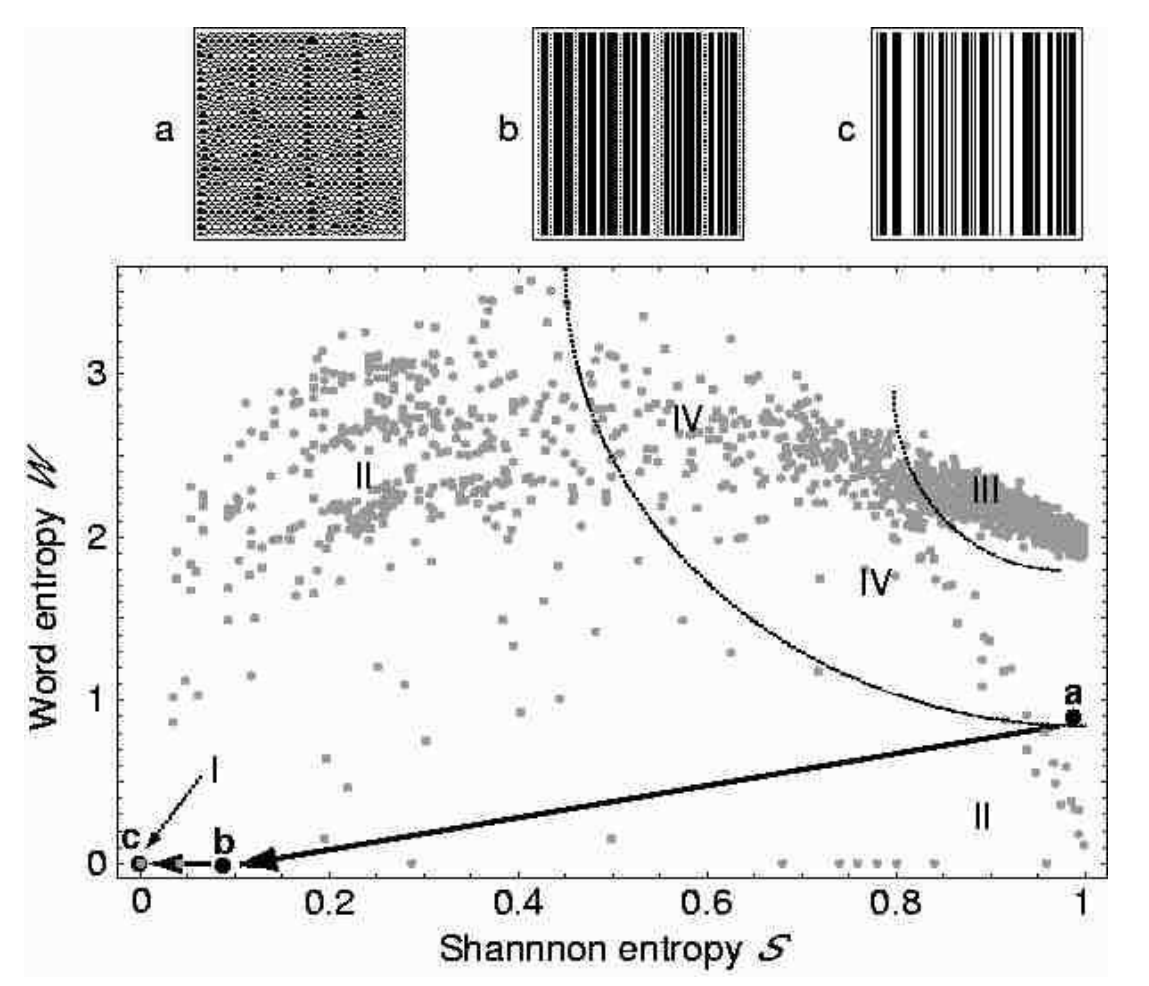

Figure 2: Behavior of $\Omega_{1}$ for elementary, i.e. $k=s=2$, CAs within the $W S$ plane as $\kappa$ is increased. The three possible neighborhood configurations correspond to the rules 54 , 108, 204 (see Tab. 1). For computation of $W$ and $S$ here and in the following the same system size as in Fig. 11 has been used $(N=500, T=1000)$ with a transient of 500 time steps being dropped. The patterns represent the three possible domains and show a selection of 100 nodes for 100 time steps for visual clarity. Note that there are no precise borders between the different regions in the WS plane. Therefore the highlighted regions reflect a tendency rather than a strict distinction between two adjacent Wolfram classes. 

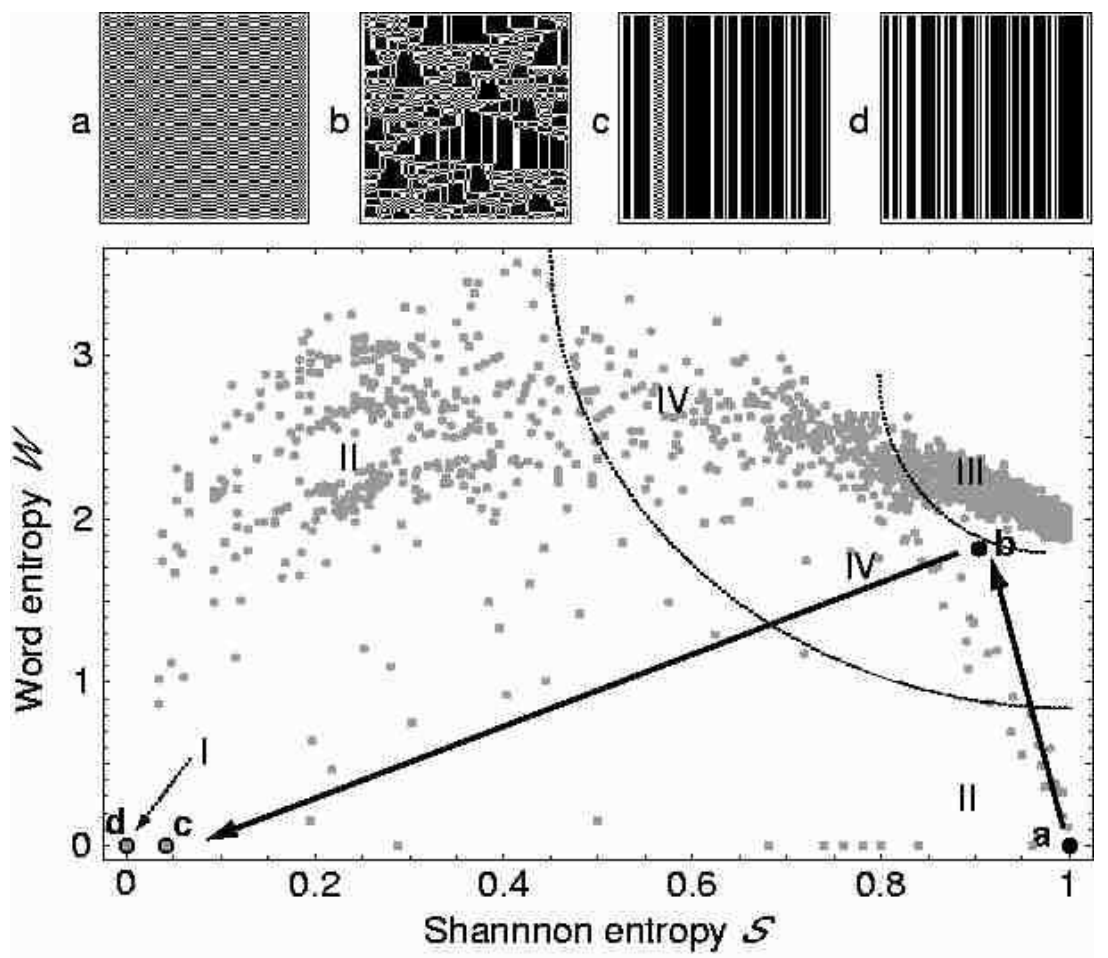

Figure 3: Behavior of $\Omega_{1}$ for $k=10$ CAs within the $W S$ plane as $\kappa$ is increased. Only for $\kappa \in[0.3,0.4[$ (b) complex patterns emerge, for all other parameters, the corresponding rules result in purely oscillatory and stationary patterns respectively. The other spatiotemporal patterns shown in the top row correspond to parameter values $\kappa \in[0.2,0.3[$ (a), $\kappa \in[0.4,0.5[$ (c) and $\kappa \in[0.5,0.6[$ (d), again for 100 nodes and 100 time steps. 

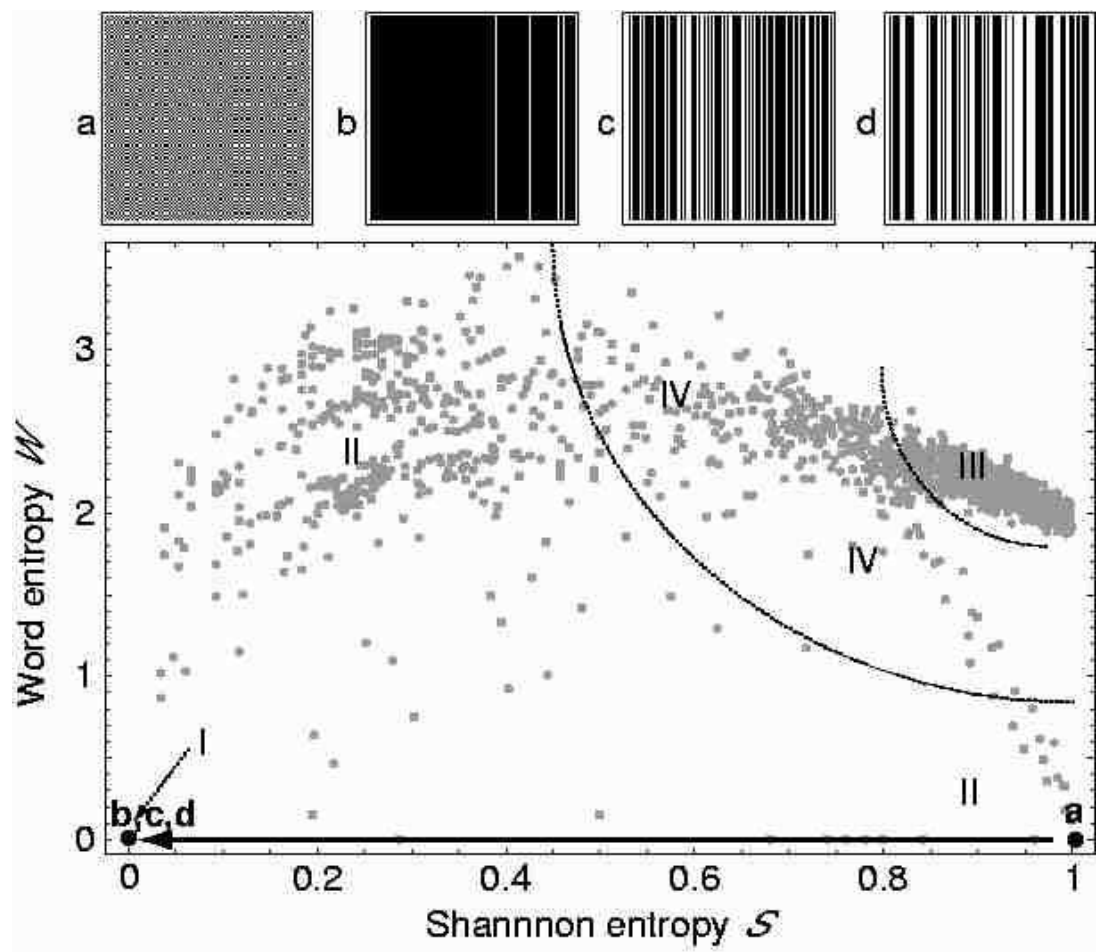

Figure 4: Behavior of $\Omega_{2}$ for elementary CAs within the $W S$ plane as $\kappa$ is increased. The corresponding rule numbers for the elementary CA are 50, 36, 76, 204 (see Tab. 11). Again, the patterns show 100 nodes for 100 time steps. All stationary patterns are mapped onto the same point in the WS plane. 


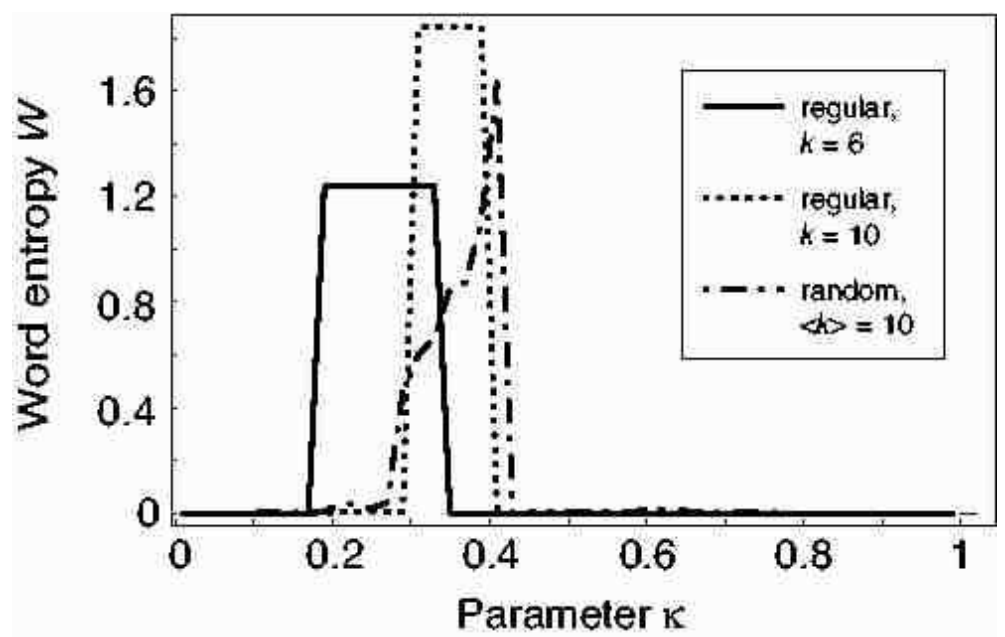

Figure 5: The word entropy $W$ against the variation of $\kappa$ for $\Omega_{1}$ for two regular graphs and an $E R$ random graph. Complex patterns prevail in the interval between $0.3<\kappa<0.4$ for the two graph types. 

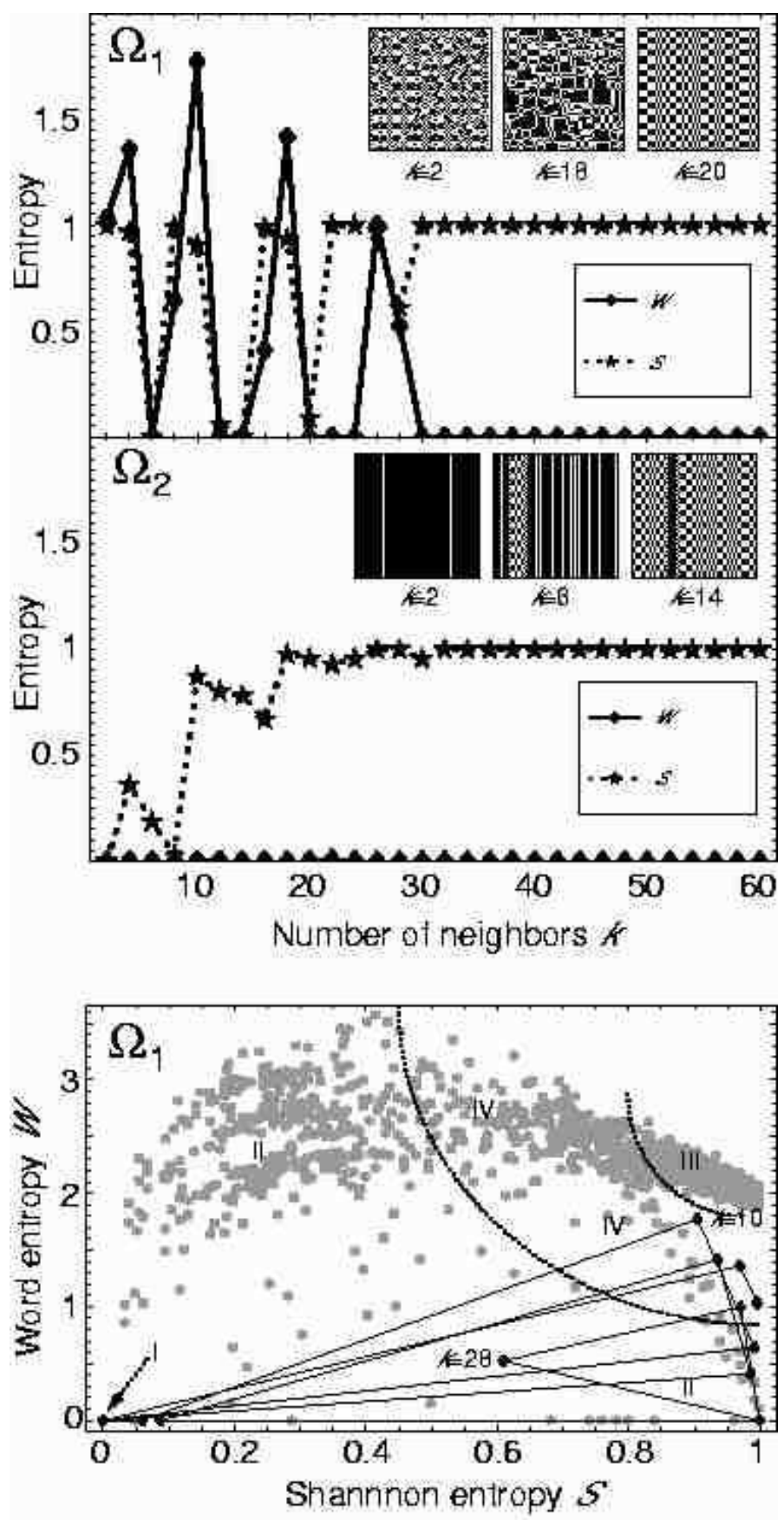

Figure 6: Word entropy (solid) and Shannon entropy (dashed) as a function of neighborhood size $k$ for $\Omega_{1}$ and $\Omega_{2}$ (top). The inset patterns show the evolution of 100 nodes for 100 time steps for three different values of $k$. The lower picture shows the trajectory of regular graphs in the WSplane under $\Omega_{1}$ as $k$ is increased. 


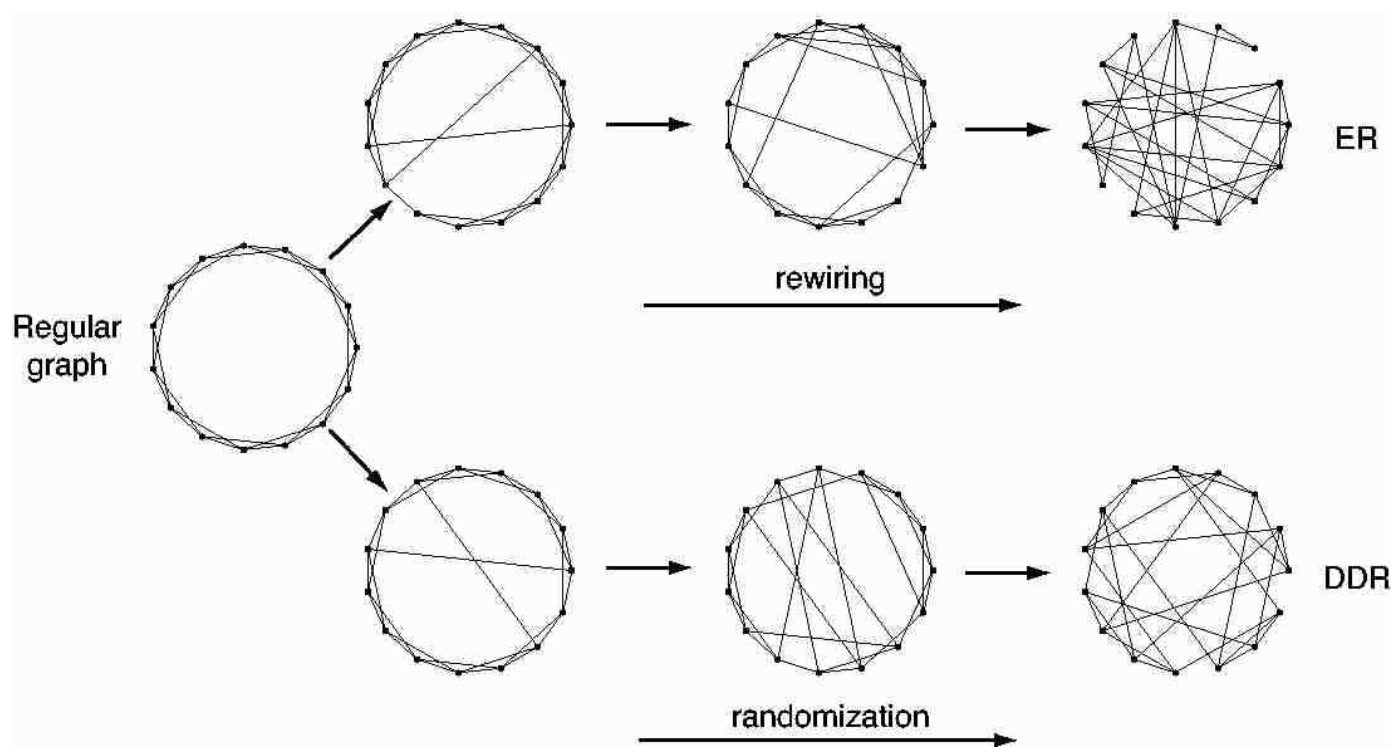

Figure 7: Rewiring (top) and randomization (bottom) of a regular graph. In the first case, the resulting degree distribution $P(k)$ is binomial and nodes with $k=2$ occur, while in the second case, the original degree distribution $P(k)=\delta_{k, 4}$ is conserved during the process. 

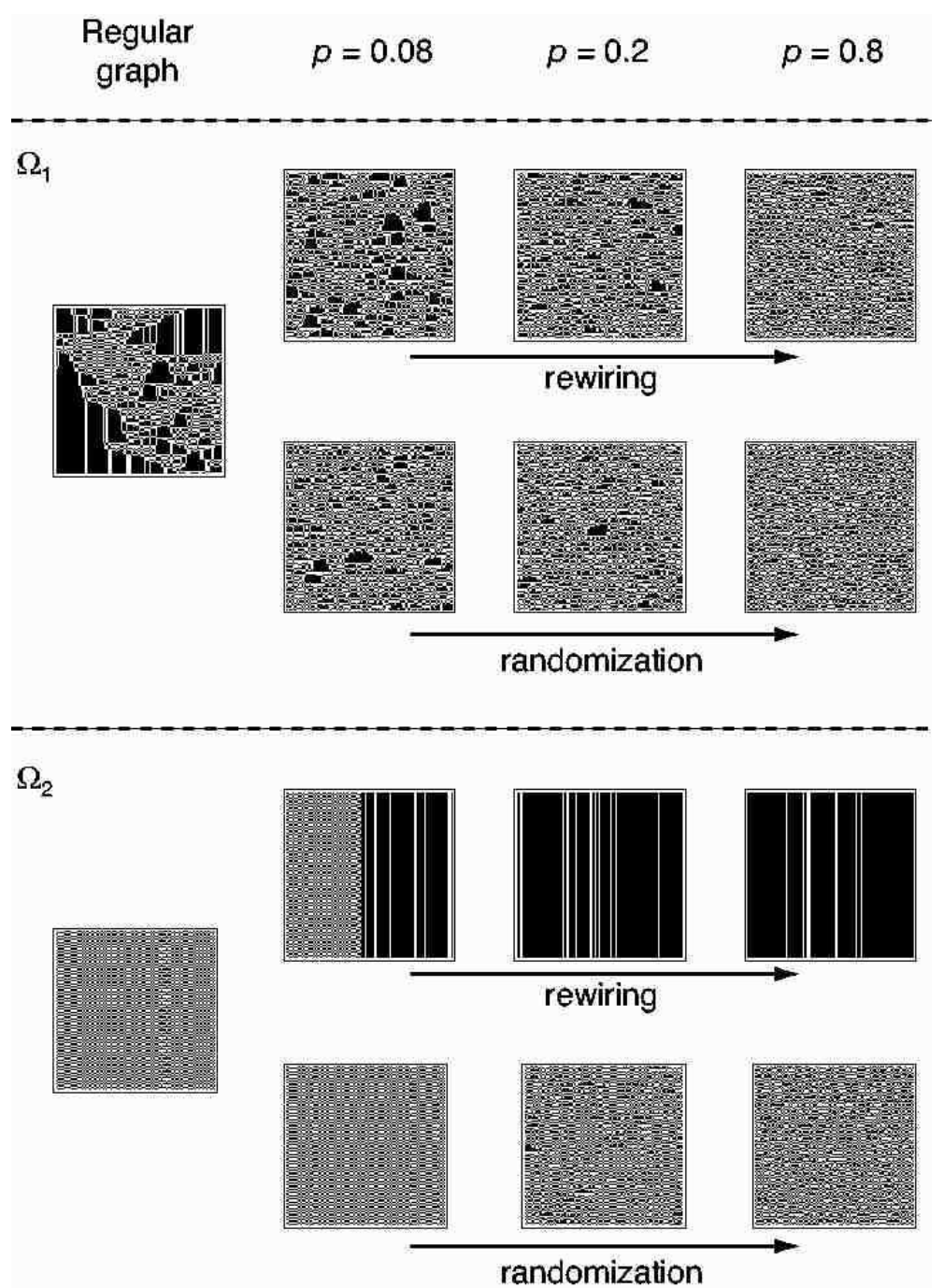

Figure 8: Spatiotemporal patterns for the different rewiring mechanisms described above for increasing rewiring and randomization depth $p$. The resulting patterns differ considerably for $\Omega_{1}$ and $\Omega_{2}$. 


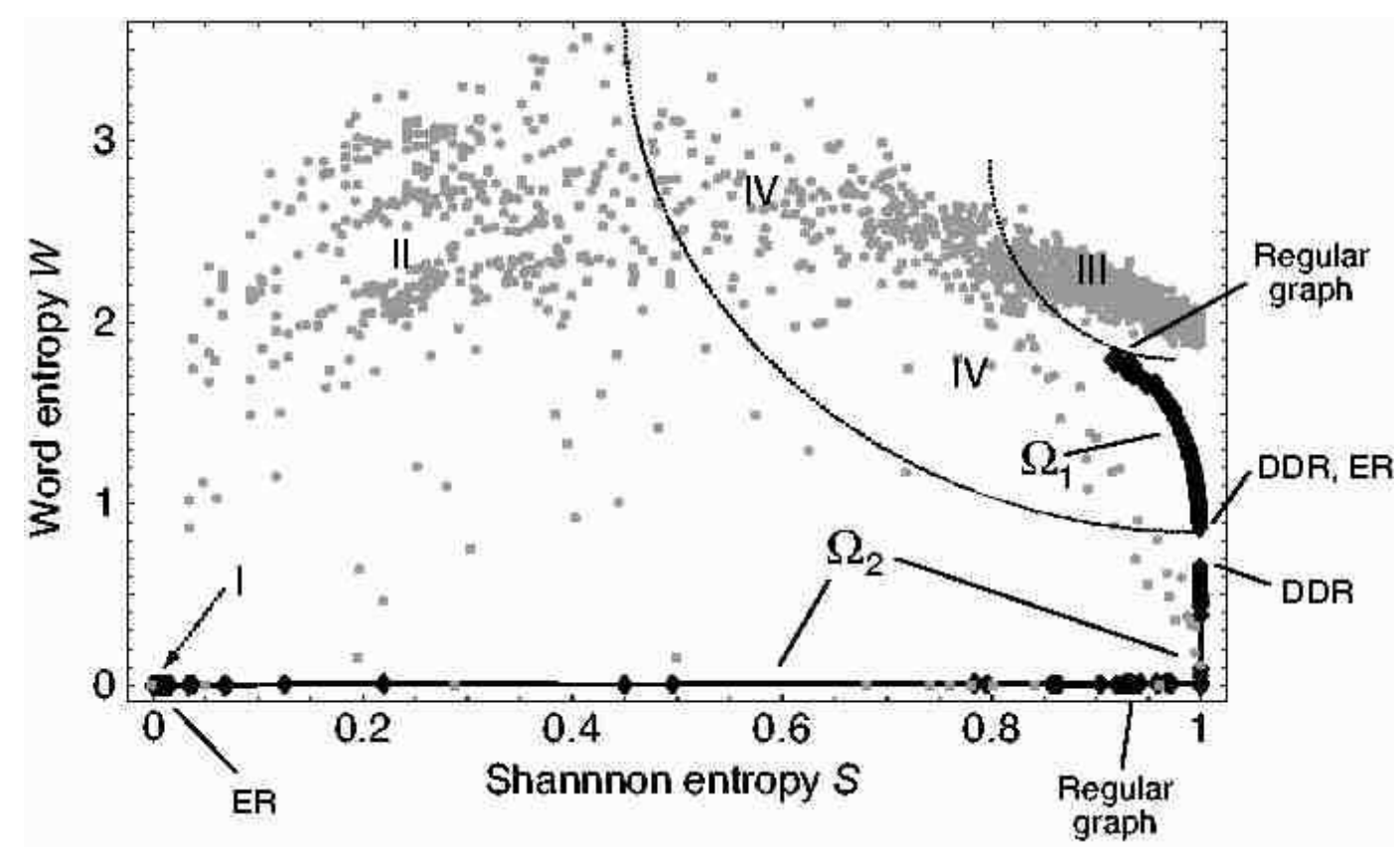

Figure 9: The four trajectories for $\Omega_{1}$ and $\Omega_{2}$ for the rewiring and randomization process of an originally regular graph with $N=500, k=10$. The two paths for $\Omega_{1}$ are nearly identical while the ones for $\Omega_{2}$ differ enormously. 


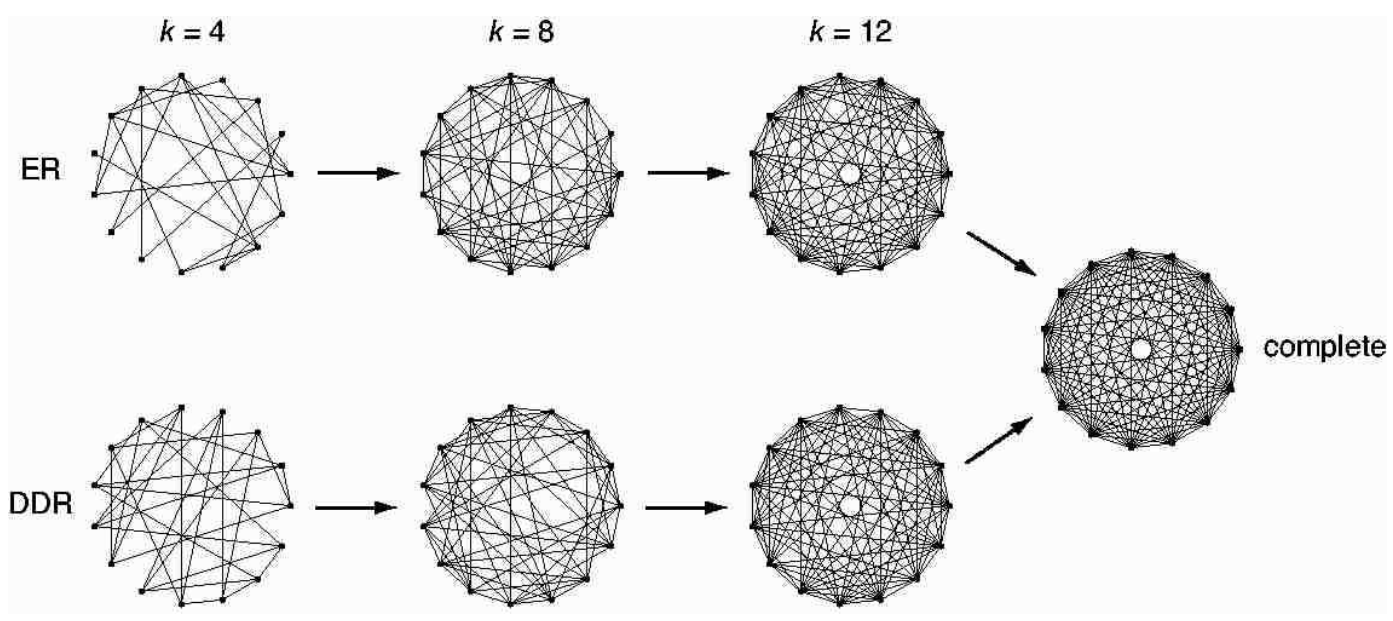

Figure 10: Increase of the degree for ER and DDR graphs. We show graphs with 15 nodes for $k=4,8,12,14$. We end up with complete graphs for both procedures. 


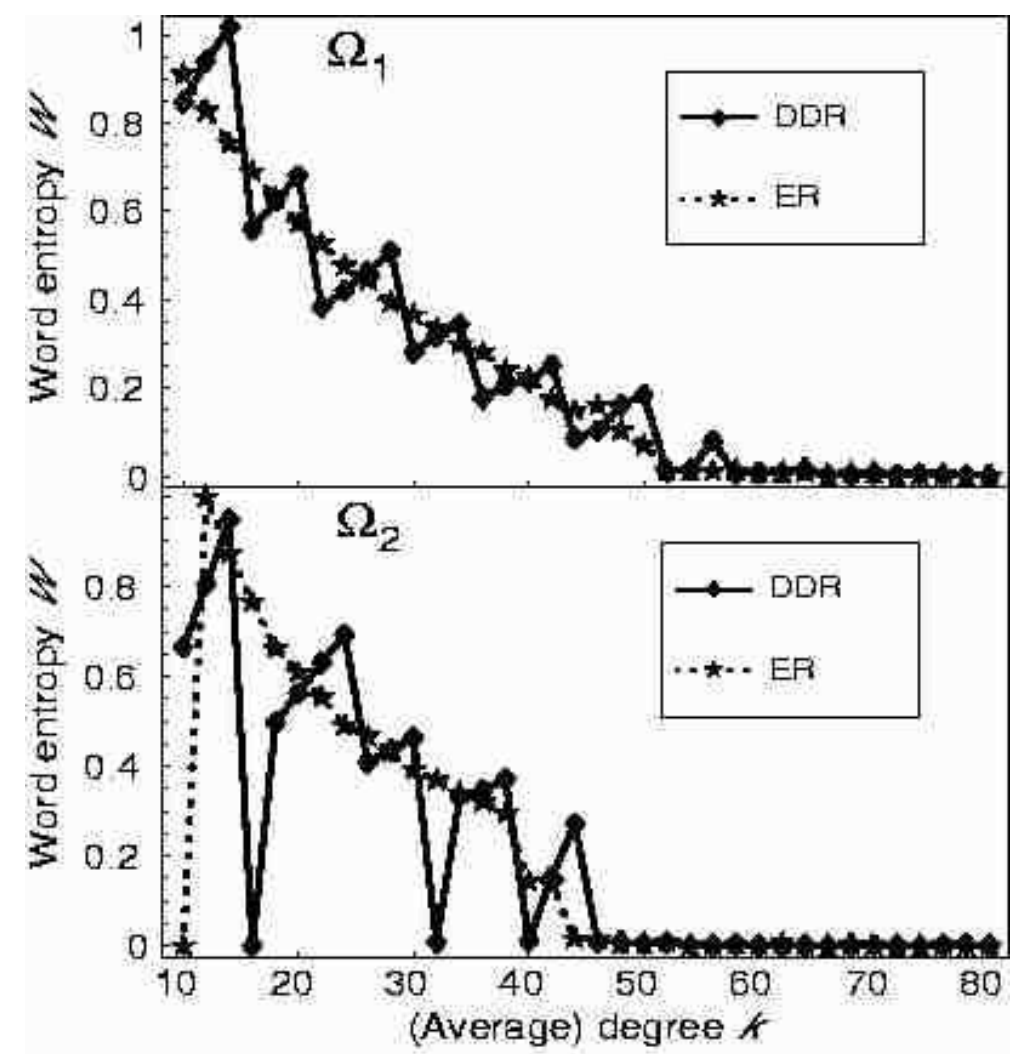

Figure 11: Word entropy $W$ for increasing $k$ for DDR (solid) and ER (dotted) graphs and both rule sets. DDR graphs show a peak structure with drops to $W=0$ for $\Omega_{2}$ (bottom). ER graphs show a similar overall behavior albeit without peaks and a smoothed curve. 


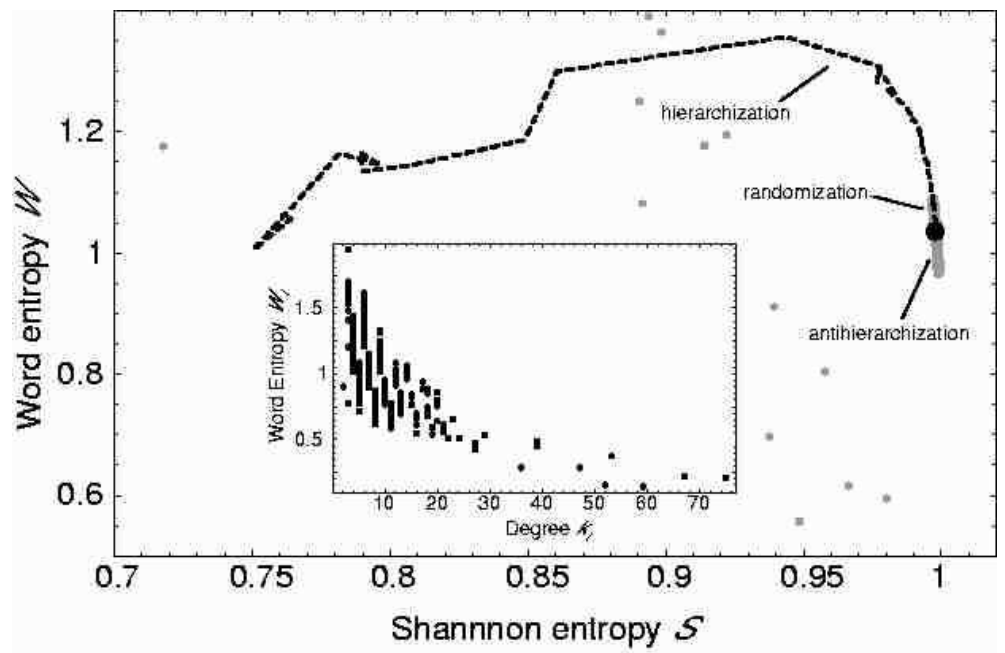

Figure 12: Effects of randomization (dashed), hierarchization (gray) and antihierarchization (gray) of a BA scale-free graph with minimal degree 4 within the $W S$ plane. The inset shows the word entropy values of 500 single nodes of the graph against their degree. Obviously, high $W_{i}$ values result from nodes with minimal degree. 


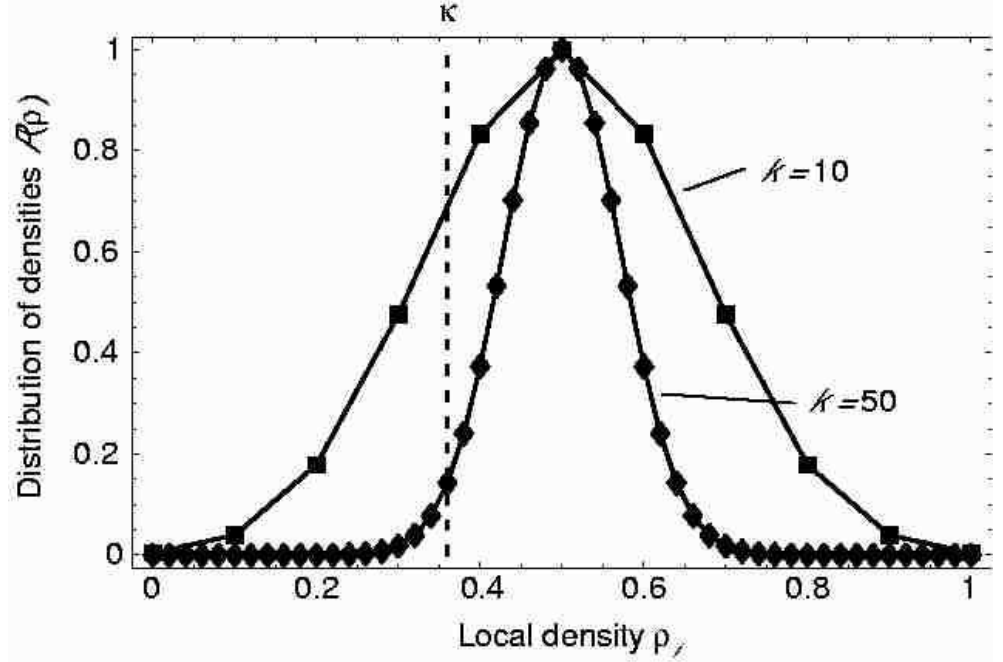

Figure 13: Scaled density distributions $P\left(\rho_{i}\right)$ for DDR graphs with $k=10$ and $k=50$. The line defined by $\kappa$ indicates the ratio of stationary to oscillatory nodes with local densities $\rho_{i}$. 

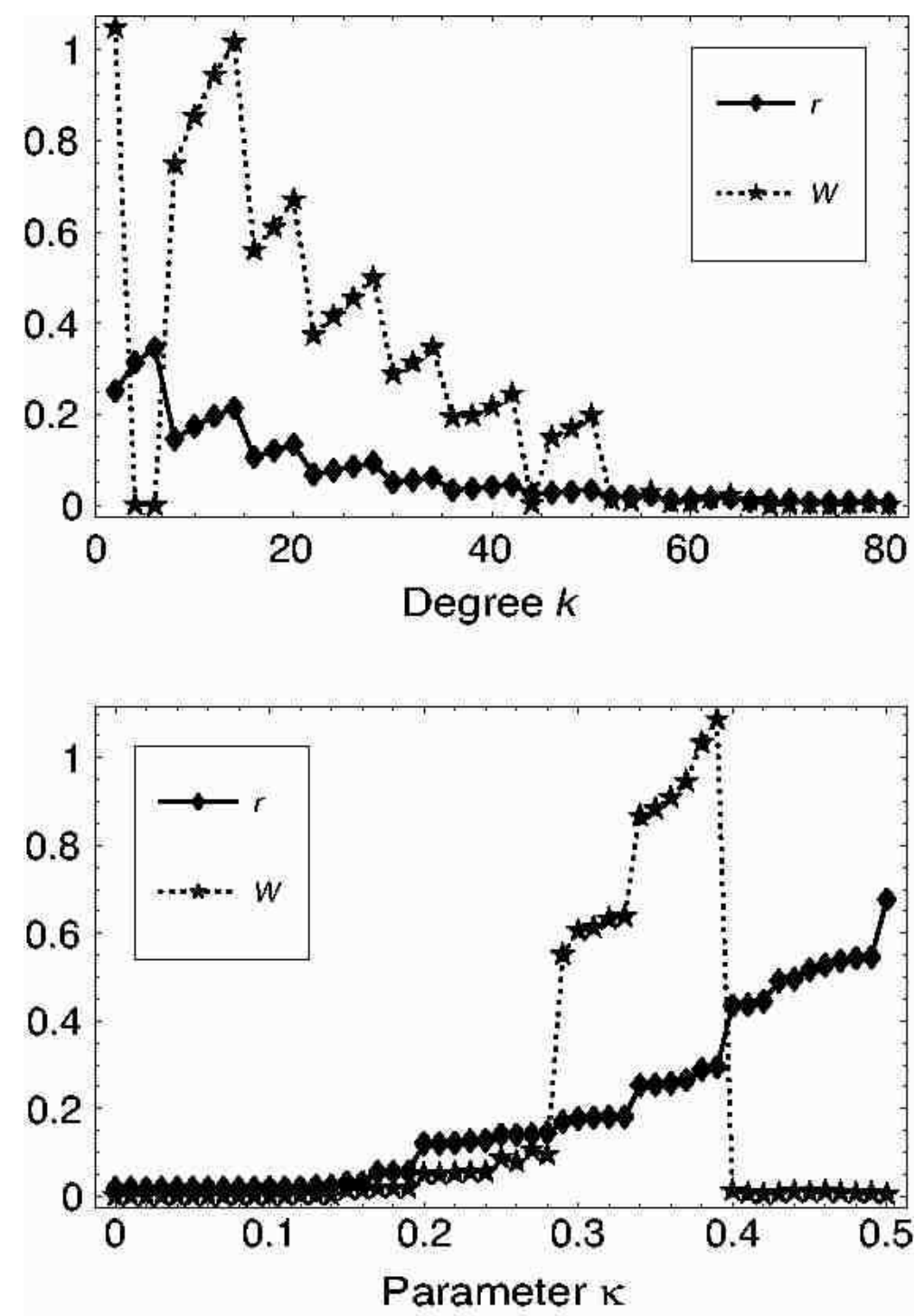

Figure 14: Fraction of constant cells $r$ compared with the word entropy $W$ for two random topologies for $\Omega_{1}$. The upper picture shows DDR graphs as $k$ is increased. The lower one shows a BA scale-free graph with minimal degree $k_{0}=5$ under variation of the parameter $\kappa$. In both figures a threshold for $r$ around 0.3 is observable, above which complex patterns disappear. 


\section{List of Tables}

1 Neighborhood mappings of rules $\Omega_{1}$ and $\Omega_{2}$ for elementary CAs. i.e. with $k=s=2$. The number 


\begin{tabular}{c|ccc|cccc}
\multicolumn{7}{c}{$\Omega_{1}$} \\
$k$ & $0 \leq \kappa<\frac{1}{2}$ & $\frac{1}{2} \leq \kappa<1$ & $\kappa=1$ & $0 \leq \kappa<\frac{1}{3}$ & $\frac{1}{3} \leq \kappa<\frac{2}{3}$ & $\frac{2}{3} \leq \kappa<1$ & $\kappa=1$ \\
\hline 000 & 0 & 0 & 0 & 0 & 0 & 0 & 0 \\
001 & $\mathbf{1}$ & 0 & 0 & $\mathbf{1}$ & 0 & 0 & 0 \\
010 & 1 & 1 & 1 & $\mathbf{0}$ & 1 & 1 & 1 \\
011 & $\mathbf{0}$ & 1 & 1 & $\mathbf{0}$ & $\mathbf{0}$ & 1 & 1 \\
100 & $\mathbf{1}$ & 0 & 0 & $\mathbf{1}$ & 0 & 0 & 0 \\
101 & $\mathbf{1}$ & $\mathbf{1}$ & 0 & $\mathbf{1}$ & $\mathbf{1}$ & 0 & 0 \\
110 & $\mathbf{0}$ & 1 & 1 & $\mathbf{0}$ & $\mathbf{0}$ & 1 & 1 \\
111 & $\mathbf{0}$ & $\mathbf{0}$ & 1 & $\mathbf{0}$ & $\mathbf{0}$ & $\mathbf{0}$ & 1 \\
\hline$\Delta$ & 54 & 108 & 204 & 50 & 36 & 76 & 204
\end{tabular}

Table 1: Neighborhood mappings of rules $\Omega_{1}$ and $\Omega_{2}$ for elementary CAs, i.e. with $k=$ $s=2$. The number of different rules is $k$ and $k+1$ for $\Omega_{1}$ and $\Omega_{2}$, respectively. If the state of the node changes, the corresponding entry is shown in bold face. In the last row, the corresponding CA rule numbers are shown. 